\title{
FURTHER RESULT OF A TYPE OF SYSTEM OF COMPLEX DIFFERENCE EQUATIONS
}

\section{MANLI LIU}

Department of Mathematics

Jinan University Guangzhou

Guangdong 510632

P. R. China

e-mail: 572996979@qq.com

\begin{abstract}
Using Nevanlinna theory of the value distribution of meromorphic functions, we investigate the form of a type of system of difference equations and extend some result of solutions of difference equations to systems of complex difference equations.
\end{abstract}

\section{Introduction}

We use the standard notation of the Nevanlinna theory of meromorphic functions (see, e.g., [1]). In addition, we denote the order of $f(z)$ by $\rho(f)$.

Recently, Laine et al. [7]; Heittokangas et al. [5]; Halburd and Korhonen [6]; Chen and Ho [2]; Chen [3]; Chiang and Feng [4] etc.

2010 Mathematics Subject Classification: 30D05, 39A10, 30D35.

Keywords and phrases: meromorphic solutions, system of difference equations, characteristic estimates, value distribution theory.

Project supported by the Natural Science Foundation of China (NO.10471065) and the Natural Science Foundation of Guangdong Province (NO.04010407).

Received November 22, 2015

(C) 2015 Scientific Advances Publishers 
investigated the existence or growth of solutions of complex difference equations, Gao [9-13] investigated the existence or growth of solutions of systems of complex difference equations, they obtain some results.

In 2005, Laine et al. [7] considered the following difference equations:

$$
\sum_{j=1}^{n} \alpha_{j}(z) f\left(z+c_{j}\right)=\frac{P(z, f(z))}{Q(z, f(z))}
$$

where the coefficients $\alpha_{j}(z)$ are non-vanishing small functions relative to $f$ and where $P, Q$ are relatively prime polynomials in $f$ over the field of small functions relative to $f$. He obtained the following theorem:

Theorem A ([7]). Suppose that $c_{1}, \cdots, c_{n}$ are distinct, non-zero complex numbers and that $f$ is a transcendental meromorphic solution of Equation (1), $q=\operatorname{deg}_{f}^{Q}>0, n=\max \{p, q\}=\max \left\{\operatorname{deg}_{f}^{P}, \operatorname{deg}_{f}^{Q}\right\}$ and that, without restricting generality, $Q$ is a monic polynomial. If there exists $\alpha \in[0, n)$ such that for all r sufficiently large,

$$
\bar{N}\left(r, \sum_{j=1}^{n} \alpha_{j}(z) f\left(z+c_{j}\right)\right) \leq \alpha \bar{N}(r+C, f(z))+S(r, f(z)),
$$

where $C=\max \left\{\left|c_{1}\right|,\left|c_{2}\right|, \ldots,\left|c_{n}\right|\right\}$, then either the order $\rho(f)=\infty$, or

$$
Q(z, f(z)) \equiv(f(z)+h(z))^{q},
$$

where $h(z)$ is a small meromorphic function.

Our question arises whether or not the assertion of Theorem A remains valid, if we replace the difference equations with system of complex difference equations. In this paper, we first consider the problem of the growth of solution of system of complex difference equations of the form: 


$$
\left\{\begin{array}{l}
\sum_{j=1}^{n} \alpha_{j}(z) w_{1}\left(z+c_{j}\right)=\frac{P_{1}\left(z, w_{2}\right)}{Q_{1}\left(z, w_{2}\right)}, \\
\sum_{j=1}^{n} \beta_{j}(z) w_{2}\left(z+c_{j}\right)=\frac{P_{2}\left(z, w_{1}\right)}{Q_{2}\left(z, w_{1}\right)},
\end{array}\right.
$$

where the coefficients $\alpha_{j}(z), \beta_{j}(z)$ are non-vanishing small functions relative to $w_{1}, w_{2}$ and $P_{1}, Q_{1}$ are relatively prime polynomials in $w_{2}$, $P_{2}, Q_{2}$ are relatively prime polynomials in $w_{1}$. We assume that $n_{1}=\max \left\{p_{1}, q_{1}\right\}, n_{2}=\max \left\{p_{2}, q_{2}\right\}, Q_{1}, Q_{2}$ are monic polynomial.

Definition 1. Let $\left(w_{1}(z), w_{2}(z)\right)$ be a set of meromorphic solutions of (2). If the component $w_{k}$ of $\left(w_{1}, w_{2}\right)$ satisfies the following condition:

$$
\limsup _{r \rightarrow \infty, r \notin I} \frac{S(r)}{T\left(r, w_{k}\right)}=0, \quad k=1,2,
$$

we say that $w_{k}$ is an admissible component of a set of solutions of (2), where $I$ is a set of finite linear measure.

Let $C=\max \left\{\left|c_{1}\right|,\left|c_{2}\right|, \ldots,\left|c_{n}\right|\right\}$. We obtain the following result:

Theorem 1. Let $\left(w_{1}(z), w_{2}(z)\right)$ be admissible meromorphic solution of (1), $\rho\left(w_{i}\right)<+\infty, i=1,2$. If there exist $\alpha, \beta \in[0, n)$ such that for all $r$ sufficiently large,

$$
\begin{aligned}
& \bar{N}\left(r, \sum_{j=1}^{n} \alpha_{j}(z) w_{1}\left(z+c_{j}\right)\right) \leq \alpha \bar{N}\left(r+C, w_{1}(z)\right)+S\left(r, w_{1}\right), \\
& \bar{N}\left(r, \sum_{j=1}^{n} \beta_{j}(z) w_{2}\left(z+c_{j}\right)\right) \leq \beta \bar{N}\left(r+C, w_{2}(z)\right)+S\left(r, w_{2}\right),
\end{aligned}
$$

then

$$
Q_{1}\left(z, w_{2}\right) \equiv\left(w_{2}(z)+h_{1}(z)\right)^{q_{1}}, \quad Q_{2}\left(z, w_{1}\right) \equiv\left(w_{1}(z)+h_{2}(z)\right)^{q_{2}},
$$

at least one of them will be true, where $h_{1}(z), h_{2}(z)$ are small meromorphic functions. 
Example 1. $\left(w_{1}(z), w_{2}(z)\right)=\left(\frac{1}{z+1}, \frac{1}{z-1}\right)$ is an admissible solution of the following system of complex difference equations:

$$
\left\{\begin{array}{l}
w_{1}(z-1)=\frac{w_{2}(z)}{w_{2}(z)+1}, \\
w_{2}(z+1)=\frac{-w_{1}(z)}{w_{1}(z)-1},
\end{array}\right.
$$

$w_{1}, w_{2}$ satisfy the conditions in Theorem $1, q_{1}=q_{2}=1$. It shows that Theorem 1 holds.

\section{Some Lemmas}

Lemma 1 ([8]). Let $w(z)$ be a meromorphic function and let $\phi$ be given by

$$
\begin{gathered}
\phi=w^{n}+a_{n-1} w^{n-1}+\cdots+a_{0}, \\
T\left(r, a_{j}\right)=S(r, w), \quad j=0, \cdots, n-1 .
\end{gathered}
$$

Then either

$$
\phi \equiv\left(w+\frac{a_{n-1}}{a_{n}}\right)^{n},
$$

or

$$
T(r, w) \leq \bar{N}\left(r, \frac{1}{\phi}\right)+\bar{N}(r, w)+S(r, w) .
$$

Lemma 2 ([7]). Let $w$ be a non-constant meromorphic function and let $P(z, w), Q(z, w)$ be two polynomials in $w$ with meromorphic coefficients small relative to $w$. If $P$ and $Q$ have no common factors of positive degree in $w$ over the field of small functions relative to $w$, then

$$
\bar{N}\left(r, \frac{1}{Q(z, w)}\right) \leq \bar{N}\left(r, \frac{P(z, w)}{Q(z, w)}\right)+S(r, w) .
$$




\section{Proof of Theorem 1}

\section{Proof. Suppose}

$$
Q_{1}\left(z, w_{2}\right) \not \equiv\left(w_{2}(z)+h_{1}(z)\right)^{q_{1}}, \quad Q_{2}\left(z, w_{1}\right) \not \equiv\left(w_{1}(z)+h_{2}(z)\right)^{q_{2}} .
$$

Then by Lemma 1, we obtain

$$
\begin{gathered}
T\left(r, w_{2}\right) \leq \bar{N}\left(r, \frac{1}{Q_{1}}\right)+\bar{N}\left(r, w_{2}\right)+S\left(r, w_{2}\right) \leq \bar{N}\left(r, \frac{P_{1}}{Q_{1}}\right)+\bar{N}\left(r, w_{2}\right)+S\left(r, w_{2}\right), \\
T\left(r, w_{2}\right)-\bar{N}\left(r, w_{2}\right) \leq \bar{N}\left(r, \frac{P_{1}}{Q_{1}}\right)+S\left(r, w_{2}\right) \\
=\bar{N}\left(r, \sum_{j=1}^{n} \alpha_{j}(z) w_{1}\left(z+c_{j}\right)\right)+S\left(r, w_{2}\right) \\
\leq \alpha \bar{N}\left(r+C, w_{1}(z)\right)+S\left(r, w_{1}\right)+S\left(r, w_{2}\right) .
\end{gathered}
$$

Similarly, we obtain

$$
T\left(r, w_{1}\right)-\bar{N}\left(r, w_{1}\right) \leq \beta \bar{N}\left(r+C, w_{2}(z)\right)+S\left(r, w_{1}\right)+S\left(r, w_{2}\right),
$$

$\rho\left(w_{i}\right)<\infty, i=1,2$ implies that

$$
S\left(r, w_{1}\left(z+c_{j}\right)\right)=S\left(r, w_{1}\right), \quad S\left(r, w_{2}\left(z+c_{j}\right)\right)=S\left(r, w_{2}\right) .
$$

Hence

$T\left(r, w_{2}\left(z+c_{j}\right)\right)-\bar{N}\left(r, w_{2}\left(z+c_{j}\right)\right) \leq \alpha \bar{N}\left(r+C, w_{1}\left(z+c_{j}\right)\right)+S\left(r, w_{1}\right)+S\left(r, w_{2}\right)$,

$T\left(r, w_{1}\left(z+c_{j}\right)\right)-\bar{N}\left(r, w_{1}\left(z+c_{j}\right)\right) \leq \beta \bar{N}\left(r+C, w_{2}\left(z+c_{j}\right)\right)+S\left(r, w_{1}\right)+S\left(r, w_{2}\right)$.

By Lemma 2 and the system (2), we have 


$$
\begin{aligned}
n_{1} T\left(r, w_{2}\right)= & T\left(r, \sum_{j=1}^{n} \alpha_{j}(z) w_{1}\left(z+c_{j}\right)\right)+S\left(r, w_{1}\right) \\
= & T\left(r, \sum_{j=1}^{n} \alpha_{j}(z) w_{1}\left(z+c_{j}\right)\right)+S\left(r, w_{1}\right) \\
& -\bar{N}\left(r, \sum_{j=1}^{n} \alpha_{j}(z) w_{1}\left(z+c_{j}\right)\right)+\bar{N}\left(r, \sum_{j=1}^{n} \alpha_{j}(z) w_{1}\left(z+c_{j}\right)\right) \\
\leq & \sum_{j=1}^{n}\left[T\left(r, w_{1}\left(z+c_{j}\right)\right)-\bar{N}\left(r, w_{1}\left(z+c_{j}\right)\right)\right] \\
& +\alpha \bar{N}\left(r+C, w_{1}(z)\right)+S\left(r, w_{1}\right) \\
\leq & \sum_{j=1}^{n} \beta \bar{N}\left(r+C, w_{2}\left(z+c_{j}\right)\right)+\alpha \bar{N}\left(r+C, w_{1}(z)\right) \\
& +S\left(r, w_{1}\right)+S\left(r, w_{2}\right) \\
& +\sum_{j=1}^{n} \beta \bar{N}\left(r+2 C, w_{2}(z)\right)+\alpha \bar{N}\left(r+C, w_{1}(z)\right) \\
& S\left(r, w_{1}\right)+S\left(r, w_{2}\right),
\end{aligned}
$$

i.e.,

$n_{1} T\left(r, w_{2}\right) \leq n \beta \bar{N}\left(r+2 C, w_{2}(z)\right)+\alpha \bar{N}\left(r+2 C, w_{1}(z)\right)+S\left(r, w_{1}\right)+S\left(r, w_{2}\right)$.

Similarly,

$n_{2} T\left(r, w_{1}\right) \leq n \alpha \bar{N}\left(r+2 C, w_{1}(z)\right)+\beta \bar{N}\left(r+2 C, w_{2}(z)\right)+S\left(r, w_{1}\right)+S\left(r, w_{2}\right)$.

Hence

$$
\left\{\begin{array}{l}
T\left(r, w_{2}\right) \leq \frac{n \beta}{n_{1}} \bar{N}\left(r+2 C, w_{2}(z)\right)+\frac{\alpha}{n_{1}} \bar{N}\left(r+2 C, w_{1}(z)\right)+S\left(r, w_{1}\right)+S\left(r, w_{2}\right), \\
T\left(r, w_{1}\right) \leq \frac{n \alpha}{n_{2}} \bar{N}\left(r+2 C, w_{1}(z)\right)+\frac{\beta}{n_{2}} \bar{N}\left(r+2 C, w_{2}(z)\right)+S\left(r, w_{1}\right)+S\left(r, w_{2}\right) .
\end{array}\right.
$$




$$
\begin{aligned}
& \int\left(r, w_{2}\right)-\bar{N}\left(r, w_{2}(z)\right) \leq \frac{n \beta}{n_{1}} \bar{N}\left(r+2 C, w_{2}(z)\right)+\frac{\alpha}{n_{1}} \bar{N}\left(r+2 C, w_{1}(z)\right) \\
& -\bar{N}\left(r, w_{2}(z)\right)+S\left(r, w_{1}\right)+S\left(r, w_{2}\right), \\
& T\left(r, w_{1}\right)-\bar{N}\left(r, w_{1}(z)\right) \leq \frac{n \alpha}{n_{2}} \bar{N}\left(r+2 C, w_{1}(z)\right)+\frac{\beta}{n_{2}} \bar{N}\left(r+2 C, w_{2}(z)\right) \\
& -\bar{N}\left(r, w_{1}(z)\right)+S\left(r, w_{1}\right)+S\left(r, w_{2}\right) . \\
& \left(T\left(r, w_{2}\right)-\bar{N}\left(r, w_{2}(z)\right) \leq \frac{\beta(n+m)}{n_{1}} \bar{N}\left(r+2 m C, w_{2}(z)\right)+\frac{m \alpha}{n_{1}} \bar{N}\left(r+2 m C, w_{1}(z)\right)\right. \\
& -m \bar{N}\left(r, w_{2}(z)\right)+S\left(r, w_{1}\right)+S\left(r, w_{2}\right), \\
& T\left(r, w_{1}\right)-\bar{N}\left(r, w_{1}(z)\right) \leq \frac{\alpha(n+m)}{n_{2}} \bar{N}\left(r+2 m C, w_{1}(z)\right)+\frac{m \beta}{n_{2}} \bar{N}\left(r+2 m C, w_{2}(z)\right) \\
& -m \bar{N}\left(r, w_{1}(z)\right)+S\left(r, w_{1}\right)+S\left(r, w_{2}\right) .
\end{aligned}
$$

We can also obtain

$$
\left\{\begin{aligned}
\bar{N}\left(r, w_{2}(z)\right) \leq \frac{\beta\left(n_{1}+m\right)}{m n_{1}} \bar{N}\left(r+2 m C, w_{2}(z)\right) & +\frac{\alpha}{n_{1}} \bar{N}\left(r+2 m C, w_{1}(z)\right) \\
& +S\left(r, w_{1}\right)+S\left(r, w_{2}\right), \\
\bar{N}\left(r, w_{1}(z)\right) \leq \frac{\alpha\left(n_{2}+m\right)}{m n_{2}} \bar{N}\left(r+2 m C, w_{1}(z)\right) & +\frac{\beta}{n_{2}} \bar{N}\left(r+2 m C, w_{2}(z)\right) \\
& +S\left(r, w_{1}\right)+S\left(r, w_{2}\right) .
\end{aligned}\right.
$$

For sufficiently large $m$, we see that

$$
\begin{aligned}
& \frac{1}{\eta_{1}}=\beta \frac{\left(n_{1}+m\right)}{m n_{1}}=\beta\left(\frac{1}{m}+\frac{1}{n_{1}}\right)<1, \\
& \frac{1}{\eta_{2}}=\alpha \frac{\left(n_{2}+m\right)}{m n_{2}}=\alpha\left(\frac{1}{m}+\frac{1}{n_{2}}\right)<1 .
\end{aligned}
$$

$\bar{N}\left(r, w_{2}(z)\right) \leq \frac{1}{\eta_{1}} \bar{N}\left(r+2 m C, w_{2}(z)\right)+\frac{\alpha}{n_{1}} \bar{N}\left(r+2 m C, w_{1}(z)\right)+S\left(r, w_{1}\right)+S\left(r, w_{2}\right)$, 


$$
\begin{aligned}
\bar{N}\left(r+2 m C, w_{2}(z)\right) \leq & \frac{1}{\eta_{1}} \bar{N}\left(r+4 m C, w_{2}(z)\right)+\frac{\alpha}{n_{1}} \bar{N}\left(r+4 m C, w_{1}(z)\right) \\
& +S\left(r+2 m C, w_{1}\right)+S\left(r+2 m C, w_{2}\right) .
\end{aligned}
$$

Hence

$$
\begin{aligned}
\bar{N}\left(r, w_{2}(z)\right) \leq & \frac{1}{\eta_{1}^{l}} \bar{N}\left(r+2 \operatorname{lm} C, w_{2}(z)\right)+\frac{1}{\eta_{1}^{l-1}} \frac{\alpha}{n_{1}} \bar{N}\left(r+2 \operatorname{lm} C, w_{1}(z)\right) \\
& +S\left(r+2(l-1) m C, w_{1}\right)+S\left(r+2(l-1) m C, w_{2}\right) .
\end{aligned}
$$

Similarly, we obtain

$$
\begin{aligned}
\bar{N}\left(r, w_{1}(z)\right) \leq & \frac{1}{\eta_{2}^{l}} \bar{N}\left(r+2 \operatorname{lm} C, w_{1}(z)\right)+\frac{1}{\eta_{2}^{l-1}} \frac{\beta}{n_{2}} \bar{N}\left(r+2 \operatorname{lm} C, w_{2}(z)\right) \\
& +S\left(r+2(l-1) m C, w_{1}\right)+S\left(r+2(l-1) m C, w_{2}\right) .
\end{aligned}
$$

But $\bar{N}\left(r+2 \operatorname{lm} C, w_{i}(z)\right)=\bar{N}\left(r, w_{i}(z)\right)+S\left(r, w_{i}\right), i=1,2$. Thus

$$
\begin{aligned}
& \bar{N}\left(r, w_{1}(z)\right) \leq \frac{1}{\eta_{2}^{l}} \bar{N}\left(r, w_{1}(z)\right)+\frac{1}{\eta_{2}^{l-1}} \bar{N}\left(r, w_{2}(z)\right)+S\left(r, w_{1}\right)+S\left(r, w_{2}\right), \\
& \bar{N}\left(r, w_{2}(z)\right) \leq \frac{1}{\eta_{1}^{l}} \bar{N}\left(r, w_{2}(z)\right)+\frac{1}{\eta_{1}^{l-1}} \bar{N}\left(r, w_{1}(z)\right)+S\left(r, w_{1}\right)+S\left(r, w_{2}\right) .
\end{aligned}
$$

Dividing through (3), (4) by $T\left(r, w_{1}(z)\right)$ and $T\left(r, w_{2}(z)\right)$, respectively,

$$
\begin{gathered}
\frac{\bar{N}\left(r, w_{1}(z)\right)}{T\left(r, w_{1}(z)\right)} \leq \frac{1}{\eta_{2}^{l}} \frac{\bar{N}\left(r, w_{1}(z)\right)}{T\left(r, w_{1}(z)\right)}+\frac{1}{\eta_{2}^{l-1}} \frac{\bar{N}\left(r, w_{2}(z)\right)}{T\left(r, w_{1}(z)\right)}+\frac{S\left(r, w_{2}\right)}{T\left(r, w_{1}\right)}+o(1), \\
\frac{\bar{N}\left(r, w_{2}(z)\right)}{T\left(r, w_{2}(z)\right)} \leq \frac{1}{\eta_{1}^{l}} \frac{\bar{N}\left(r, w_{2}(z)\right)}{T\left(r, w_{2}(z)\right)}+\frac{1}{\eta_{1}^{l-1}} \frac{\bar{N}\left(r, w_{1}(z)\right)}{T\left(r, w_{2}(z)\right)}+\frac{S\left(r, w_{1}\right)}{T\left(r, w_{2}\right)}+o(1) .
\end{gathered}
$$

Combining (5) and (6), we have 
$\left(\eta_{1}^{l}-1+o(1)\right)\left(\eta_{2}^{l}-1+o(1)\right) \frac{\bar{N}\left(r, w_{2}(z)\right)}{T\left(r, w_{2}(z)\right)} \frac{\bar{N}\left(r, w_{1}(z)\right)}{T\left(r, w_{1}(z)\right)}$

$\leq \eta_{1} \eta_{2} \frac{\bar{N}\left(r, w_{2}(z)\right)}{T\left(r, w_{2}(z)\right)} \frac{\bar{N}\left(r, w_{1}(z)\right)}{T\left(r, w_{1}(z)\right)}+\frac{\bar{N}\left(r, w_{2}(z)\right)}{T\left(r, w_{1}(z)\right)} \frac{S\left(r, w_{1}(z)\right)}{T\left(r, w_{2}(z)\right)}+\frac{\bar{N}\left(r, w_{1}(z)\right)}{T\left(r, w_{2}(z)\right)} \frac{S\left(r, w_{2}(z)\right)}{T\left(r, w_{1}(z)\right)}$

According to $w_{1}, w_{2}$ are admissible, taking the upper limit as $r \rightarrow \infty$, we obtain

$$
\begin{aligned}
&\left(\eta_{1}^{l}-1\right)\left(\eta_{2}^{l}-1\right) \lim \sup _{r \rightarrow \infty} \frac{\bar{N}\left(r, w_{2}(z)\right)}{T\left(r, w_{2}(z)\right)} \frac{\bar{N}\left(r, w_{1}(z)\right)}{T\left(r, w_{1}(z)\right)} \\
& \leq\left(\eta_{1} \eta_{2}\right) \lim \sup _{r \rightarrow \infty} \frac{\bar{N}\left(r, w_{2}(z)\right)}{T\left(r, w_{2}(z)\right)} \frac{\bar{N}\left(r, w_{1}(z)\right)}{T\left(r, w_{1}(z)\right)},
\end{aligned}
$$

that is,

$$
\left(\eta_{1}^{l}-1\right)\left(\eta_{2}^{l}-1\right) \leq \eta_{1} \eta_{2}
$$

This is a contradiction to the $\eta_{i}>1, i=1,2, l>1$.

This implies that

$$
Q_{1}\left(z, w_{2}\right) \equiv\left(w_{2}(z)+h_{1}(z)\right)^{q_{1}}, \quad Q_{2}\left(z, w_{1}\right) \equiv\left(w_{1}(z)+h_{2}(z)\right)^{q_{2}}
$$

at least one of them will be true.

\section{References}

[1] Hongxun Yi and C. C. Yang, Theory of the Uniqueness of Meromorphic Functions (in Chinese), Science Press, Beijing, 1995.

[2] Zongxuan Chen and Shon Kwang Ho, On the growth of solutions of a class of higher order differential equation, Acta Mathematica Scientia, Ser. B 24(1) (2004), 52-60.

[3] Zongxuan Chen, Growth and zeros of meromorphic solution of some linear difference equations, Journal of Mathematical Analysis and Applications 373(1) (2010), $235-241$.

[4] Y. M. Chiang and S. J. Feng, On the Nevanlinna characteristic of $f(z+\eta)$ and difference equations in the complex plane, Ramanujan Journal 16(1) (2008), 105-129. 
[5] J. Heittokangas, R. Korhonen, I. Laine, J. Rieppo and K. Tohge, Complex difference equations of Malmquist type, Computational Methods and Function Theory 1(1) (2001), 27-39.

[6] R. G. Halburd and R. J. Korhonen, Difference analogue of the lemma on the logarithmic derivative with applications to difference equations, Journal of Mathematical Analysis and Applications 314(2) (2006), 477-487.

[7] I. Laine, J. Rieppo and H. Silvennoinen, Remarks on complex difference equations, Computational Methods and Function Theory 5(1) (2005), 77-88.

[8] G. Weissenborn, On the theorem of Tumura and Clunie, The Bulletin of the London Mathematical Society 18(4) (1986), 371-373.

[9] Lingyun Gao, Systems of complex difference equations of Malmquist type, Acta Mathematica Sinica 55(2) (2012), 293-300.

[10] Lingyun Gao, Estimates of $N$-function and $m$-function of meromorphic solutions of systems of complex difference equations, Acta Mathematica Scientia 32B(4) (2012), 1495-1502.

[11] Lingyun Gao, Solutions of complex higher-order difference equations, Acta Mathematica Sinica 56(4) (2013), 451-458.

[12] Lingyun Gao, On meromorphic solutions of a type of difference equations, Chinese Ann. Math. 35A(2) (2014), 193-202.

[13] Lingyun Gao, The growth order of solutions of systems of complex difference equations, Acta Mathematica Scientia 33B(3) (2013), 814-820.

[14] R. Goldstein, Some results on factorisation of meromorphic functions, London Math. Soc. 4(2) (1971), 357-364.

[15] R. Goldstein, On meromorphic solutions of certain functional equations, Aequationes Math. 17(1) (1978), 112-157. 